Review Article

\title{
Involvement of Reduced Microbial Diversity in Inflammatory Bowel Disease
}

\author{
Dawei Gong, ${ }^{1}$ Xiaojie Gong, ${ }^{2}$ Lili Wang, ${ }^{1}$ Xinjuan Yu, ${ }^{1}$ and Quanjiang Dong ${ }^{1}$ \\ ${ }^{1}$ Department of Central Laboratories and Gastroenterology, Qingdao Municipal Hospital, School of Medicine, \\ Qingdao University, Qingdao 266071, China \\ ${ }^{2}$ Department of Emergency Surgery, The Fifth People's Hospital of Jinan, Jinan 250022, China
}

Correspondence should be addressed to Quanjiang Dong; jiangacer@126.com

Received 3 August 2016; Revised 19 October 2016; Accepted 17 November 2016

Academic Editor: Tamar Ringel-Kulka

Copyright (C) 2016 Dawei Gong et al. This is an open access article distributed under the Creative Commons Attribution License, which permits unrestricted use, distribution, and reproduction in any medium, provided the original work is properly cited.

\begin{abstract}
A considerable number of studies have been conducted to study the microbial profiles in inflammatory conditions. A common phenomenon in inflammatory bowel disease (IBD) is the reduction of the diversity of microbiota, which demonstrates that microbial diversity negatively correlates with disease severity in IBD. Increased microbial diversity is known to occur in disease remission. Species diversity plays an important role in maintaining the stability of the intestinal ecosystem as well as normal ecological function. A reduction in microbial diversity corresponds to a decrease in the stability of the ecosystem and can impair ecological function. Fecal microbiota transplantation (FMT), probiotics, and prebiotics, which aim to modulate the microbiota and restore its normal diversity, have been shown to be clinically efficacious. In this study, we hypothesized that a reduction in microbial diversity could play a role in the development of IBD.
\end{abstract}

\section{Introduction}

Inflammatory bowel disease (IBD) is a chronic disease, and the two main forms of IBD are ulcerative colitis (UC) and Crohn's disease (CD). IBD has been shown to be associated with increased morbidity in developed countries and developing countries that are gradually adopting a more modern lifestyle. IBD has been reported to be caused by a multitude of factors such as genetics, environment, immune system, and gut microbiota. Although its exact pathogenesis remains unclear, dysbiosis of the microbiota in the intestinal tract is widely accepted to initiate or promote intestinal inflammation [1-3].

An increasing number of studies have used noncultured $16 S$ rRNA sequencing technology to reveal intestinal microbial profiles in IBD and healthy controls. Some meaningful features were found. The gastrointestinal tract contains several hundred microbial species [4-7], most of which belong to the Firmicutes, Bacteroidetes, Proteobacteria, and Actinobacteria phyla $[8,9]$. A balanced microbial population in the human gut could benefit the human body by providing nutrients, maintaining immune homeostasis, and granting niche protection. However, recent studies have demonstrated a dysbiosis in IBD patients. CD patients had significantly higher populations of Streptococcus and Enterococcus and lower populations of Coprococcus, Roseburia, Faecalibacterium, and Ruminococcus compared with healthy controls [10]. Furthermore, the abundance of Bacteroides, Enterococcus, Blautia, and Escherichia-Shigella genera was significantly increased in patients with UC, and the abundance of Coprococcus decreased compared with healthy controls [10]. Andoh et al. found that the abundance of Clostridium was decreased in patients with active UC and inactive/active CD patients, whereas the abundance of Bacteroides significantly increased in the patients with CD [11]. Although the altered microbial profiles did not show any consistent results across many studies, a common feature, that is, reduced microbiota diversity, emerged in all patients with IBD [7, 12-16].

The diversity of microbiota is closely associated with disease conditions. In a study on microbiota signatures in eczema, Nylund et al. found that the severity of eczema was inversely correlated with the microbial diversity $(r=-0.54$; $P=0.005)$, indicating that the lower the microbial diversity, the higher the severity of eczema [17]. Additionally, the 
microbial diversity increased with improvement of symptoms in eczema [17]. A similar relationship was found between the microbial diversity and disease severity in human IBD [18]. Russell et al. treated neonatal mice with vancomycin and reported that this resulted in a reduction in microbial diversity accompanied by an increase in the severity of asthma [19]. The level of diversity is also related to the response to therapy in patients. For example, children with UC who responded to corticosteroid medication were reported to have a higher diversity than nonresponders [20].

Little attention has been paid to the role of reduced microbial diversity in the function of the intestinal ecosystem. This review intends to reveal the relationship between a reduction in diversity and IBD from a biological perspective.

\section{Reduced Microbial Diversity in IBD}

A previous study compared the intestinal microbial flora between patients with active $\mathrm{CD}$ and healthy controls using $16 S$ rRNA gene sequencing. The mean Shannon diversity was lower in CD patients than in the healthy controls [21]. Similar to these findings, in a multicenter study, fecal samples collected from 161 patients with CD and 121 healthy individuals found that the active/inactive CD patients had a significantly lower Shannon diversity than the healthy controls [22]. Sha et al. analyzed the diversity of fecal microbiota in IBD patients compared with healthy controls using denaturing gradient gel electrophoresis. The participants included patients with UC, patients with $\mathrm{CD}$, and healthy controls. The microbial diversity was remarkably lower in IBD patients than in the healthy control group, and the reduced microbial diversity was more obvious in the active UC and active CD groups [13]. Additionally, studies of microbial profiles from UC patients revealed that these patients have lower fecal microbial diversity and lower abundance of major anaerobic bacteria (Bacteroides and Clostridium subcluster XIVa) compared with healthy controls [23]. In another study, microarray hybridization was used to study the characteristics of the microbiota from healthy controls and children hospitalized with severe UC. The richness, evenness, and biodiversity of the gut microbiota were all significantly reduced in children with UC compared with healthy controls; furthermore, a reduction in the abundance of Clostridium and an increase in the class Gammaproteobacteria were found [20].

The precise causal relationship between the inflammatory state and a reduction in bacterial diversity remains unknown. To determine whether defects in the mucosal barrier and bacterial dysbiosis are inherently abrogated in the terminal ileum (TI) of patients with UC (where inflammation is absent), the TI was biopsied from patients with CD and UC and from healthy controls without IBD [24]. Despite the absence of ileitis, UC patients displayed ileal barrier depletion and a reduction in the $\alpha$-diversity compared with pediatric patients without IBD [24]. In a study on mice, when antibiotics were used to deplete the gut microbiota, a reduction in diversity and mild gut inflammation occurred [25]. In another study, microbial profiles of patients with $\mathrm{CD}$, their healthy siblings, and unrelated healthy individuals were sequenced and analyzed. Healthy siblings who were at a higher risk of developing
CD had lower core microbial diversity than low-risk healthy controls (although the microbial diversity in the siblings was higher than that in the CD patients), suggesting that the loss of core microbial diversity may be a fundamental step in the pathogenesis of CD [26]. Interestingly, the hygiene hypothesis suggests that improved hygiene conditions may lead to a reduction in the intestinal microbial diversity and that it may in turn be responsible for the development of IBD [27].

\section{Attempt to Restore Normal Diversity to Alleviate IBD}

FMT involves the transfer of fecal suspension from a healthy donor to the intestinal tract of a recipient, modulating imbalanced gut microbiota and restoring normal diversity and bacterial composition of the intestine. FMT therapy is currently used in treatment of intestinal inflammation [28].

FMT has received extended attention in the treatment of IBD [29-31]. Many studies have revealed that FMT can induce the remission of some IBD patients. Moayyedi et al. conducted FMT in patients with active UC in a randomized controlled trial. In their study, patients with UC were examined using flexible sigmoidoscopy at the start of the study and were randomly assigned to groups that received an enema of either $50 \mathrm{~mL}$ of FMT (from healthy anonymous donors, $n=38$ ) or $50 \mathrm{~mL}$ of placebo (water, $n=37$ ) once per week for six weeks. The primary outcome was remission of $\mathrm{UC}$, defined as a Mayo score $\leq 2$ with an endoscopic Mayo score of 0 at week 7 of treatment. In their study, nine of the patients who received FMT (24\%) and 2 who received placebo (5\%) achieved remission $(P=0.03)$ [32]. Additionally, the fecal samples of patients receiving FMT had greater microbial diversity compared with baseline [33]. A systematic review and meta-analysis from a large multigroup sample after FMT therapy found that remission after FMT could reach 36.2\% [34]. A subgroup analysis revealed that the clinical remission of the UC group and the remission rate of the CD group reached $22 \%$ and $60.5 \%$, respectively [34]. FMT has also been reported to have the best efficacy in treating patients when first diagnosed [32].

Clostridium difficile infection (CDI) is caused by toxinproducing $C$. difficile and features a range of symptoms from mild diarrhea to potentially lethal conditions such as pseudomembranous colitis. Low et al. found that the microbial diversity was lower in patients with CDI than in healthy controls, and alterations in the composition included a reduction in the abundance of Firmicutes and Bacteroidetes as well as an increased abundance of Proteobacteria [35]. Notably, a study on CDI reported that the reduction in microbial diversity occurs prior to the occurrence of CDI, emphasizing the promoting effect of reduced diversity on intestinal inflammation [36]. Antibiotic therapy for CDI cannot achieve the desired effect and can induce recurrent Clostridium difficile infection (rCDI) [37]. In other studies, increased microbiota diversity could be achieved in recipients who achieved remission after FMT $[35,38,39]$. Accordingly, when FMT was used to treat CDI, a significant curative effect could be achieved $[28,40]$. van Nood et al. studied the efficacy of FMT in rCDI compared with vancomycin to treat recurrent infection. They found 
a significantly higher rate in the resolution of $C$. difficileassociated diarrhea in the FMT group with the resolution of symptoms in nearly all patients in the FMT group [40]. After the FMT procedure, the recipients showed increased microbial diversity at levels similar to the microbial features of the healthy donor [40]. To investigate the eradication of C. difficile and changes in the microbiome following FMT in children with and without IBD, 8 children (5 with IBD and 3 without IBD) with a history of recurrent CDI $(\geq 3$ recurrences) received FMT via colonoscopy. All 8 children showed the resolution of CDI symptoms and eradication of $C$. difficile at 10-20 weeks and 6 months after the administration of FMT [41]. There was also an increase in the intestinal microbial diversity after FMT therapy [41]. Three systematic reviews and a meta-analysis on CDI treated with FMT showed an average curative rate of approximately 90\% [4244]. Furthermore, a controlled trial revealed that the curative rate of rCDI treated with FMT could reach $81 \%$ [40]. FMT therapy is associated with fewer adverse reactions $[45,46]$. The most successful application of FMT was reported in the treatment of CDI [47]. Although the pathogenesis of CDI is significantly different from that of IBD, the microbiota of patients with both conditions have been found to be similar, and both conditions have shown successful outcomes following FMT treatment, especially in CDI, indicating that the normal intestinal microbiota plays an indispensable role in human homeostasis and that reduced diversity of the microbiota could cause intestinal inflammation.

\section{Potential Factors Contributing to a Reduction in Microbial Diversity}

The gastrointestinal tract environment may be considered as an ecosystem. It includes the mucosal epithelium, mucus layer, bacteria, viruses, funguses, parasites, and archaea [4]. The health of the gastrointestinal ecosystem is primarily represented by its microbial diversity. A balanced ecosystem represents normal ecological function and gut health. However, the ecological environment is disturbed in IBD, resulting in reduced microbial diversity. Therefore, the question arises: what are the factors that induce a reduction in microbial diversity? Knowing the exact mechanism could help reduce the risks associated with reduced diversity and provide therapeutic strategies to treat it.

4.1. Role of Antibiotics, Diet, and Environments. Antibiotics are widely used and can lead to significant changes in the composition of the intestinal microbial flora [48-51]. Jakobsson et al. studied the effects of metronidazole and clarithromycin on intestinal microbiota and found that the drugs caused a significant diversity reduction [52]. The antibiotics disrupted the microbiota and caused antibiotic-associated diarrhea $[53,54]$. Diet is also responsible for altering the composition of the intestinal microbiota [55]. In a recent study, Sonnenburg et al. reported that a diet low in dietary fiber could decrease the diversity of intestinal microbiota in mice and that a diet that is persistently low in dietary fiber resulted in a progressive loss of microbial diversity in subsequent generations of mice. Conversely, a diet rich in dietary fiber helped maintain high intestinal microbiota diversity in mice of all generations [56]. This phenomenon may explain the higher morbidity of IBD in Western countries, which tend to have high-fat low-fiber diets. Recently, a systematic review focusing on the impact of diet on gut microbiota also indicated that a fiber-rich diet could elevate microbial diversity [57]. Environmental factors can also significantly impact the microbial profile [58]; the microbial profiles in Malawian and Venezuelan populations are more diverse than those of adults and children residing in the US [59].

4.2. Role of Genetics, Immune System, and Mucus. To study the potential role of the chromatin remodeler CHD1 in shaping the gut microbiome of Drosophila melanogaster, Sebald et al. performed deep $16 S$ rRNA gene sequencing of gut microbiota from $C h d 1^{-/-}$and control Chd1 ${ }^{W T / W T}$ flies, which carried a wild-type Chd1 rescue transgene in a Chd1deficient genetic background. Interestingly, principal coordinate analysis revealed clear distinctions between the microbiota of mutant and wild-type flies. The $C h d 1^{-/-}$flies had a significantly lower microbial diversity than the Chd1 $1^{W T / W T}$ controls [60]. This implies the genetic function in shaping the structure and composition of microbiota. Lim et al. investigated the effect of heritability and host genetics on gut microbiota and metabolic syndrome and reported that the patients with metabolic syndrome had lower microbial diversity than healthy individuals. The microbial profiles were significantly associated with the special host genotype [61, $62]$. The role of adaptive immunity on the gut microbiota was investigated in a mouse model to determine the role of immunity in the regulation of gut microflora. The microbiota of immunodeficient $\mathrm{Ragl}^{-/-}$mice and wild-type mice housed in the same conditions were analyzed using $16 \mathrm{~S}$ rRNA sequencing [63]. The results demonstrated that $\mathrm{Rag1}^{-/-}$ mice had distinct microbiota and had a higher increase in microbial diversity with increasing age compared to wildtype mice [63]. To a certain extent, this study may imply that immune enhancement could suppress microbial diversity. Furthermore, the human intestinal tract surface is covered with a mucus layer that prevents intimate interaction between the intestinal epithelium and bacteria $[64,65]$. The colon mucus layer in humans consists of an inner layer and an outer layer. The viscosity of the outer layer is low, making it easy to penetrate by bacteria. However, the inner layer of the mucus is viscous and sterile [66]. The mucus plays an indispensable role in maintaining homeostasis in the human intestinal tract. During inflammation, the mucus layer is impaired and infiltrated by microbes [64]. MUC2, which is primarily produced by goblet cells, is a major component of the colonic mucus layer [66, 67]. In a MUC2-deficient mouse model, mice lacking MUC2 were found to develop spontaneous colitis $[68,69]$. Mucin is an energy source for intestinal microbiota and could regulate the composition of intestinal microflora [70]. Bel et al. studied $\mathrm{TMF}^{-/-}$mice lacking TMF/ARA160 and found that these mice produce thick and uniform colonic mucus. The microbial features of $\mathrm{TMF}^{-/-}$knockout mice and wild-type mice were analyzed and the Shannon diversity index was higher in the former 
than in the latter group [71]. When dextran sulfate sodium (DSS) was used to induce colitis in two mice groups, the results showed that the knockout mice (with higher diversity) exhibited an attenuated response to DSS compared to their wild-type counterparts. Both groups of mice were cohoused for 4 weeks, and the diversity of microbiota was higher in the cohoused wild-type mice than in the wild-type mice housed alone; additionally, the cohoused wild-type mice had diminished susceptibility to induced colitis [71].

Many studies have already reported the association between these factors and IBD. Interestingly, these factors, which contribute a reduction in microbial diversity, are also risk factors for developing IBD [72-76].

\section{Mechanism of Reduction in Diversity}

Biodiversity plays an important role in maintaining a balanced ecosystem by contributing to the stability of an ecosystem as well as ecological functioning [77-79]. A high diversity provides the ecosystem with strong stability, which is defined as the ability to resist disturbance and to maintain normal ecological function [80]. When diversity is lost, the stability of an ecosystem decreases, which means that it is more susceptible to even minor assaults. This may explain why the lower gut microbial diversity observed in Western populations is associated with a higher morbidity rate of IBD. Consequently, IBD patients who achieve remission but still have lower microbial diversity are more likely to experience relapse.

Biodiversity is positively correlated with ecological function, and ecological function can maintain a balanced state when the biodiversity is at a certain level for functional redundancy $[81,82]$. When the balance is disrupted, the biodiversity reduces and the normal function of the ecosystem is transformed to another state, which is undesirable [83]. To determine the precise dysfunction in the intestinal ecosystem of patients with IBD, Morgan et al. studied microbial metabolism in IBD patients and healthy subjects using shotgun metagenomes. They identified major shifts in metabolic pathways, including an increase in the occurrence of oxidative stress pathways and a decrease in basic metabolism and short-chain fatty acid (SCFA) production [84]. The microbiome of patients with ileal CD is known to exhibit heightened virulence and secretion pathways [84]. One study compared the metagenome of the microbiota between children with active $C D$ with a reduced diversity and altered stool microbial composition and healthy controls. Modules that were more abundant in the CD group included ubiquinone and lipopolysaccharide (LPS) biosynthesis, as well as the twin-arginine translocation system; sulfur reduction was also noted, whereas key processes such as fatty acid biosynthesis were overrepresented in the controls [21]. LPS can activate the toll-like receptor 4 (TLR-4) signaling pathway to induce inflammation [85]. Duboc et al. compared stool samples from IBD patients and healthy subjects to study the impact of dysbiosis in IBD on metabolism to bile acids and inflammation of the epithelium. The microbiota of IBD patients exhibited impaired deconjugation, transformation, and desulfation to bile acids, demonstrating an increase in the conjugated bile acid rates and fecal 3-OH-sulfated bile acids as well as a decrease in the secondary bile acids. The results also showed that secondary bile acids could lead to anti-inflammation activities [86].

The reduction in diversity, accompanied by alterations in the microbial structure, could induce a disturbance in normal intestinal ecological function, which is harmful to the host [84]. These phenomena suggest that a reduction in microbial diversity has a far-reaching impact on the gut ecosystem, deteriorating the gut environment and inducing intestinal inflammation. Future research should further investigate the relationship between microbial diversity and intestinal health to promote the clinical remission of intestinal inflammation via biotherapy.

\section{Conclusion}

The diversity of microbiota contributes to the stability of the intestinal ecosystem and its ecological function. A loss of diversity could initiate an inflammatory reaction and promote the development of inflammatory disease. Bacterial therapy, including FMT, probiotics, and prebiotics, has obtained curative efficacy accompanied by an improvement in diversity [87-92]. The restoration of normal microbiota diversity represents a promising prospect in curing corresponding inflammatory disease.

\section{Abbreviations \\ IBD: Inflammatory bowel disease \\ FMT: Fecal microbiota transplantation \\ UC: Ulcerative colitis \\ CD: Crohn's disease \\ TI: Terminal ileum \\ CDI: Clostridium difficile infection \\ rCDI: Recurrent Clostridium difficile infection \\ SCFA: Short-chain fatty acid \\ LPS: Lipopolysaccharide \\ TLR-4: Toll-like receptor 4 .}

\section{Competing Interests}

The authors declare that they have no competing interests.

\section{References}

[1] B. Chassaing and A. T. Gewirtz, "Gut microbiota, low-grade inflammation, and metabolic syndrome," Toxicologic Pathology, vol. 42, no. 1, pp. 49-53, 2014.

[2] S. Brugman, K.-Y. Liu, D. Lindenbergh-Kortleve et al., "Oxazolone-induced enterocolitis in zebrafish depends on the composition of the intestinal microbiota," Gastroenterology, vol. 137, no. 5, pp. 1757el-1767el, 2009.

[3] P. M. Munyaka, N. Eissa, C. N. Bernstein, E. Khafipour, and J. E. Ghia, "Antepartum antibiotic treatment increases offspring susceptibility to experimental colitis: a role of the gut microbiota," PLoS ONE, vol. 10, no. 11, Article ID e0142536, 2015.

[4] F. Sommer and F. Bäckhed, "The gut microbiota-masters of host development and physiology," Nature Reviews Microbiology, vol. 11, no. 4, pp. 227-238, 2013. 
[5] Human Microbiome Project C, "Structure, function and diversity of the healthy human microbiome," Nature, vol. 486, pp. 207-214, 2012.

[6] J. J. Faith, J. L. Guruge, M. Charbonneau et al., "The long-term stability of the human gut microbiota," Science, vol. 341, no. 6141, Article ID 1237439, 2013.

[7] J. Qin, R. Li, J. Raes et al., "A human gut microbial gene catalogue established by metagenomic sequencing," Nature, vol. 464, no. 7285, pp. 59-65, 2010.

[8] L. Rigottier-Gois, "Dysbiosis in inflammatory bowel diseases: the oxygen hypothesis," ISME Journal, vol. 7, no. 7, pp. 12561261, 2013.

[9] P. De Cruz, S. Kang, J. Wagner et al., "Association between specific mucosa-associated microbiota in Crohn's disease at the time of resection and subsequent disease recurrence: a pilot study," Journal of Gastroenterology and Hepatology, vol. 30, no. 2, pp. 268-278, 2015.

[10] L. Chen, W. Wang, R. Zhou et al., "Characteristics of fecal and mucosa-associated microbiota in Chinese patients with inflammatory bowel disease," Medicine, vol. 93, no. 8, article e51, 2014.

[11] A. Andoh, H. Imaeda, T. Aomatsu et al., "Comparison of the fecal microbiota profiles between ulcerative colitis and Crohn's disease using terminal restriction fragment length polymorphism analysis," Journal of Gastroenterology, vol. 46, no. 4, pp. 479-486, 2011.

[12] K. Ray, "IBD: Gut microbiota in IBD goes viral," Nature Reviews Gastroenterology and Hepatology, vol. 12, article no. 122, 2015.

[13] S. Sha, B. Xu, X. Wang et al., "The biodiversity and composition of the dominant fecal microbiota in patients with inflammatory bowel disease," Diagnostic Microbiology and Infectious Disease, vol. 75, no. 3, pp. 245-251, 2013.

[14] J. Dicksved, J. Halfvarson, M. Rosenquist et al., "Molecular analysis of the gut microbiota of identical twins with Crohn's disease," ISME Journal, vol. 2, no. 7, pp. 716-727, 2008.

[15] C. Manichanh, L. Rigottier-Gois, E. Bonnaud et al., "Reduced diversity of faecal microbiota in Crohn's disease revealed by a metagenomic approach," Gut, vol. 55, no. 2, pp. 205-211, 2006.

[16] S. J. Ott, M. Musfeldt, D. F. Wenderoth et al., "Reduction in diversity of the colonic mucosa associated bacterial microflora in patients with active inflammatory bowel disease," Gut, vol. 53, no. 5, pp. 685-693, 2004.

[17] L. Nylund, M. Nermes, E. Isolauri, S. Salminen, W. M. de Vos, and R. Satokari, "Severity of atopic disease inversely correlates with intestinal microbiota diversity and butyrate-producing bacteria," Allergy, vol. 70, no. 2, pp. 241-244, 2015.

[18] E. Papa, M. Docktor, C. Smillie et al., "Non-invasive mapping of the gastrointestinal microbiota identifies children with inflammatory bowel disease," PLoS ONE, vol. 7, no. 6, Article ID e39242, 2012.

[19] S. L. Russell, M. J. Gold, M. Hartmann et al., "Early life antibiotic-driven changes in microbiota enhance susceptibility to allergic asthma," EMBO Reports, vol. 13, no. 5, pp. 440-447, 2012.

[20] S. Michail, M. Durbin, D. Turner et al., "Alterations in the gut microbiome of children with severe ulcerative colitis," Inflammatory Bowel Diseases, vol. 18, no. 10, pp. 1799-1808, 2012.

[21] C. Quince, U. Z. Ijaz, N. Loman et al., "Extensive modulation of the fecal metagenome in children with Crohn's disease during exclusive enteral nutrition," American Journal of Gastroenterology, vol. 110, no. 12, pp. 1718-1729, 2015.

[22] A. Andoh, H. Kuzuoka, T. Tsujikawa et al., "Multicenter analysis of fecal microbiota profiles in Japanese patients with Crohn's disease," Journal of Gastroenterology, vol. 47, no. 12, pp. 12981307, 2012.

[23] H. Nemoto, K. Kataoka, H. Ishikawa et al., "Reduced diversity and imbalance of fecal microbiota in patients with ulcerative colitis," Digestive Diseases and Sciences, vol. 57, no. 11, pp. 29552964, 2012.

[24] M. Alipour, D. Zaidi, R. Valcheva et al., "Mucosal barrier depletion and loss of bacterial diversity are primary abnormalities in paediatric ulcerative colitis," Journal of Crohn's and Colitis, vol. 10, no. 4, pp. 462-471, 2016.

[25] L. Grasa, L. Abecia, R. Forcén et al., "Antibiotic-induced depletion of murine microbiota induces mild inflammation and changes in toll-like receptor patterns and intestinal motility," Microbial Ecology, vol. 70, no. 3, pp. 835-848, 2015.

[26] C. Hedin, C. J. van der Gast, G. B. Rogers et al., "Siblings of patients with Crohn's disease exhibit a biologically relevant dysbiosis in mucosal microbial metacommunities," Gut, vol. 65, no. 6, pp. 944-953, 2016.

[27] G. A. W. Rook, "99th Dahlem conference on infection, inflammation and chronic inflammatory disorders: darwinian medicine and the 'hygiene' or 'old friends' hypothesis," Clinical and Experimental Immunology, vol. 160, no. 1, pp. 70-79, 2010.

[28] T. J. Borody and J. Campbell, "Fecal microbiota transplantation. Techniques, applications, and issues," Gastroenterology Clinics of North America, vol. 41, no. 4, pp. 781-803, 2012.

[29] S. Kunde, A. Pham, S. Bonczyk et al., "Safety, tolerability, and clinical response after fecal transplantation in children and young adults with ulcerative colitis," Journal of Pediatric Gastroenterology and Nutrition, vol. 56, no. 6, pp. 597-601, 2013.

[30] T. J. Borody, E. F. Warren, S. Leis, R. Surace, and O. Ashman, "Treatment of ulcerative colitis using fecal bacteriotherapy," Journal of Clinical Gastroenterology, vol. 37, no. 1, pp. 42-47, 2003.

[31] L. P. Smits, K. E. C. Bouter, W. M. De Vos, T. J. Borody, and M. Nieuwdorp, "Therapeutic potential of fecal microbiota transplantation," Gastroenterology, vol. 145, no. 5, pp. 946-953, 2013.

[32] P. Moayyedi, M. G. Surette, P. T. Kim et al., "Fecal microbiota transplantation induces remission in patients with active ulcerative colitis in a randomized controlled trial," Gastroenterology, vol. 149, no. 1, Article ID 59708, pp. 102-109, 2015.

[33] Y. Shi, Y. Dong, W. Huang et al., "Fecal microbiota transplantation for ulcerative colitis: a systematic review and metaanalysis," PLOS ONE, vol. 11, no. 6, Article ID e0157259, 2016.

[34] R. J. Colman and D. T. Rubin, "Fecal microbiota transplantation as therapy for inflammatory bowel disease: a systematic review and meta-analysis," Journal of Crohn's and Colitis, vol. 8, no. 12, pp. 1569-1581, 2014.

[35] D. E. Low, D. Shahinas, M. Silverman et al., "Toward an understanding of changes in diversity associated with fecal microbiome transplantation based on 16s rRNA gene deep sequencing," mBio, vol. 3, no. 5, Article ID e00338-12, 2012.

[36] C. Vincent, D. A. Stephens, V. G. Loo et al., "Reductions in intestinal Clostridiales precede the development of nosocomial Clostridium difficile infection," Microbiome, vol. 1, no. 1, article 18, 2013.

[37] L. V. McFarland, "Alternative treatments for Clostridium difficile disease: what really works?" Journal of Medical Microbiology, vol. 54, no. 2, pp. 101-111, 2005.

[38] R. A. Britton and V. B. Young, "Role of the intestinal microbiota in resistance to colonization by Clostridium difficile," Gastroenterology, vol. 146, no. 6, pp. 1547-1553, 2014. 
[39] M. Rupnik, M. H. Wilcox, and D. N. Gerding, "Clostridium difficile infection: new developments in epidemiology and pathogenesis," Nature Reviews Microbiology, vol. 7, no. 7, pp. 526-536, 2009.

[40] E. van Nood, A. Vrieze, M. Nieuwdorp et al., "Duodenal infusion of donor feces for recurrent Clostridium difficile," The New England Journal of Medicine, vol. 368, no. 5, pp. 407-415, 2013.

[41] S. K. Hourigan, L. A. Chen, Z. Grigoryan et al., "Microbiome changes associated with sustained eradication of Clostridium difficile after single faecal microbiota transplantation in children with and without inflammatory bowel disease," Alimentary Pharmacology and Therapeutics, vol. 42, no. 6, pp. 741-752, 2015.

[42] Z. Kassam, C. H. Lee, Y. Yuan, and R. H. Hunt, "Fecal microbiota transplantation for clostridium difficile infection: systematic review and meta-analysis," American Journal of Gastroenterology, vol. 108, no. 4, pp. 500-508, 2013.

[43] N. G. Rossen, J. K. MacDonald, E. M. de Vries et al., "Fecal microbiota transplantation as novel therapy in gastroenterology: a systematic review," World Journal of Gastroenterology, vol. 21, no. 17, pp. 5359-5371, 2015.

[44] Y.-T. Li, H.-F. Cai, Z.-H. Wang, J. Xu, and J.-Y. Fang, "Systematic review with meta-analysis: long-term outcomes of faecal microbiota transplantation for Clostridium difficile infection," Alimentary Pharmacology and Therapeutics, vol. 43, no. 4, pp. 445-457, 2016.

[45] J. Landy, H. O. Al-Hassi, S. D. McLaughlin et al., "Review article: faecal transplantation therapy for gastrointestinal disease," Alimentary Pharmacology and Therapeutics, vol. 34, no. 4, pp. 409-415, 2011.

[46] T. Spector and R. Knight, "Authors' reply to Mawer and Wilcox and Mullish and Williams," British Medical Journal, vol. 351, Article ID h6132, 2015.

[47] T. J. Borody and A. Khoruts, "Fecal microbiota transplantation and emerging applications," Nature Reviews Gastroenterology and Hepatology, vol. 9, no. 2, pp. 88-96, 2012.

[48] C. G. Buffie, I. Jarchum, M. Equinda et al., "Profound alterations of intestinal microbiota following a single dose of clindamycin results in sustained susceptibility to Clostridium difficileinduced colitis," Infection and Immunity, vol. 80, no. 1, pp. 62-73, 2012.

[49] J. Yin, X.-X. Zhang, B. Wu, and Q. Xian, "Metagenomic insights into tetracycline effects on microbial community and antibiotic resistance of mouse gut," Ecotoxicology, vol. 24, no. 10, pp. 21252132, 2015.

[50] G. Dubourg, J. C. Lagier, C. Robert et al., "Culturomics and pyrosequencing evidence of the reduction in gut microbiota diversity in patients with broad-spectrum antibiotics," International Journal of Antimicrobial Agents, vol. 44, no. 2, pp. 117-124, 2014.

[51] L. Dethlefsen, S. Huse, M. L. Sogin, and D. A. Relman, "The pervasive effects of an antibiotic on the human gut microbiota, as revealed by deep 16s rRNA sequencing," PLoS Biology, vol. 6, no. 11, article no. e280, pp. 2383-2400, 2008.

[52] H. E. Jakobsson, C. Jernberg, A. F. Andersson, M. SjölundKarlsson, J. K. Jansson, and L. Engstrand, "Short-term antibiotic treatment has differing long-term impacts on the human throat and gut microbiome," PLoS ONE, vol. 5, no. 3, Article ID e9836, 2010.

[53] P. A. Johanesen, K. E. Mackin, M. L. Hutton et al., "Disruption of the gut microbiome: clostridium difficile infection and the threat of antibiotic resistance," Genes, vol. 6, no. 4, pp. 13471360, 2015.
[54] J. Momper, Y. Mulugeta, and G. Burckart, "Failed pediatric drug development trials," Clinical Pharmacology \& Therapeutics, vol. 98, no. 3, pp. 245-251, 2015.

[55] E. P. Halmos, C. T. Christophersen, A. R. Bird, S. J. Shepherd, P. R. Gibson, and J. G. Muir, "Diets that differ in their FODMAP content alter the colonic luminal microenvironment," Gut, vol. 64, no. 1, pp. 93-100, 2015.

[56] E. D. Sonnenburg, S. A. Smits, M. Tikhonov, S. K. Higginbottom, N. S. Wingreen, and J. L. Sonnenburg, "Diet-induced extinctions in the gut microbiota compound over generations," Nature, vol. 529, no. 7585, pp. 212-215, 2016.

[57] H. L. Simpson and B. J. Campbell, "Review article: dietary fibre-microbiota interactions," Alimentary Pharmacology and Therapeutics, vol. 42, no. 2, pp. 158-179, 2015.

[58] P. J. Turnbaugh, M. Hamady, T. Yatsunenko et al., "A core gut microbiome in obese and lean twins," Nature, vol. 457, no. 7228, pp. 480-484, 2009.

[59] T. Yatsunenko, F. E. Rey, M. J. Manary et al., "Human gut microbiome viewed across age and geography," Nature, vol. 486, no. 7402, pp. 222-227, 2012.

[60] J. Sebald, M. Willi, I. Schoberleitner et al., "Impact of the chromatin remodeling factor $\mathrm{CHD} 1$ on gut microbiome composition of Drosophila melanogaster," PLoS ONE, vol. 11, no. 4, Article ID e0153476, 2016.

[61] M. Y. Lim, H. J. You, H. S. Yoon et al., “The effect of heritability and host genetics on the gut microbiota and metabolic syndrome," Gut, 2016.

[62] D. N. Frank, C. E. Robertson, C. M. Hamm et al., "Disease phenotype and genotype are associated with shifts in intestinalassociated microbiota in inflammatory bowel diseases," Inflammatory Bowel Diseases, vol. 17, no. 1, pp. 179-184, 2011.

[63] H. Zhang, J. B. Sparks, S. V. Karyala, R. Settlage, and X. M. Luo, "Host adaptive immunity alters gut microbiota," ISME Journal, vol. 9, no. 3, pp. 770-781, 2015.

[64] M. E. V. Johansson, J. K. Gustafsson, J. Holmen-Larsson et al., "Bacteria penetrate the normally impenetrable inner colon mucus layer in both murine colitis models and patients with ulcerative colitis," Gut, vol. 63, no. 2, pp. 281-291, 2014.

[65] L. A. Van Der Waaij, H. J. M. Harmsen, M. Madjipour et al., "Bacterial population analysis of human colon and terminal ileum biopsies with 16S rRNA-based fluorescent probes: commensal bacteria live in suspension and have no direct contact with epithelial cells," Inflammatory Bowel Diseases, vol. 11, no. 10, pp. 865-871, 2005.

[66] M. E. V. Johansson, M. Phillipson, J. Petersson, A. Velcich, L. Holm, and G. C. Hansson, "The inner of the two Muc2 mucindependent mucus layers in colon is devoid of bacteria," Proceedings of the National Academy of Sciences of the United States of America, vol. 105, no. 39, pp. 15064-15069, 2008.

[67] M. E. V. Johansson, J. M. H. Larsson, and G. C. Hansson, "The two mucus layers of colon are organized by the MUC2 mucin, whereas the outer layer is a legislator of host-microbial interactions," Proceedings of the National Academy of Sciences of the United States of America, vol. 108, supplement 1, pp. 4659-4665, 2011.

[68] C. K. Heazlewood, M. C. Cook, R. Eri et al., "Aberrant mucin assembly in mice causes endoplasmic reticulum stress and spontaneous inflammation resembling ulcerative colitis," PLoS Medicine, vol. 5, no. 3, article no. e54, 2008.

[69] M. Van der Sluis, B. A. E. De Koning, A. C. J. M. De Bruijn et al., "Muc2-deficient mice spontaneously develop colitis, indicating 
that MUC2 is critical for colonic protection," Gastroenterology, vol. 131, no. 1, pp. 117-129, 2006.

[70] S. Etzold and N. Juge, "Structural insights into bacterial recognition of intestinal mucins," Current Opinion in Structural Biology, vol. 28, no. 1, pp. 23-31, 2014.

[71] S. Bel, Y. Elkis, H. Elifantz et al., "Reprogrammed and transmissible intestinal microbiota confer diminished susceptibility to induced colitis in $\mathrm{TMF}^{-/-}$mice," Proceedings of the National Academy of Sciences of the United States of America, vol. 111, no. 13, pp. 4964-4969, 2014.

[72] A. N. Ananthakrishnan, H. Khalili, G. G. Konijeti et al., "A prospective study of long-term intake of dietary fiber and risk of Crohn's disease and ulcerative colitis," Gastroenterology, vol. 145, no. 5, pp. 970-977, 2013.

[73] X. Li, J. Sundquist, K. Hemminki, and K. Sundquist, "Risk of inflammatory bowel disease in first- and second-generation immigrants in Sweden: a nationwide follow-up study," Inflammatory Bowel Diseases, vol. 17, no. 8, pp. 1784-1791, 2011.

[74] S. Y. Shaw, J. F. Blanchard, and C. N. Bernstein, "Association between the use of antibiotics in the first year of life and pediatric inflammatory bowel disease," The American Journal of Gastroenterology, vol. 105, no. 12, pp. 2687-2692, 2010.

[75] D. K. Amre, S. D’Souza, K. Morgan et al., "Imbalances in dietary consumption of fatty acids, vegetables, and fruits are associated with risk for crohn's disease in children," American Journal of Gastroenterology, vol. 102, no. 9, pp. 2016-2025, 2007.

[76] C. S. J. Probert, V. Jayanthi, D. Pinder, A. C. Wicks, and J. F. Mayberry, "Epidemiological study of ulcerative proctocolitis in Indian migrants and the indigenous population of Leicestershire," Gut, vol. 33, no. 5, pp. 687-693, 1992.

[77] Y. Feng, "Species diversity and managed ecosystem stability," Chinese Journal of Applied Ecology, vol. 14, no. 6, pp. 853-857, 2003.

[78] A. S. Mori, T. Furukawa, and T. Sasaki, "Response diversity determines the resilience of ecosystems to environmental change," Biological Reviews, vol. 88, no. 2, pp. 349-364, 2013.

[79] T. H. Oliver, M. S. Heard, N. J. Isaac et al., "Biodiversity and resilience of ecosystem functions," Trends in Ecology \& Evolution, vol. 30, no. 11, pp. 673-684, 2015.

[80] F. Isbell, D. Craven, J. Connolly et al., "Biodiversity increases the resistance of ecosystem productivity to climate extremes," Nature, vol. 526, no. 7574, pp. 574-577, 2015.

[81] M. Loreau, "Biodiversity and ecosystem functioning: the mystery of the deep sea," Current Biology, vol. 18, no. 3, pp. R126R128, 2008.

[82] P. Balvanera, A. B. Pfisterer, N. Buchmann et al., "Quantifying the evidence for biodiversity effects on ecosystem functioning and services," Ecology Letters, vol. 9, no. 10, pp. 1146-1156, 2006.

[83] M. Scheffer, S. Carpenter, J. A. Foley, C. Folke, and B. Walker, "Catastrophic shifts in ecosystems," Nature, vol. 413, no. 6856, pp. 591-596, 2001.

[84] X. C. Morgan, T. L. Tickle, H. Sokol et al., "Dysfunction of the intestinal microbiome in inflammatory bowel disease and treatment," Genome Biology, vol. 13, article no. R79, 2012.

[85] A. Poltorak, X. He, I. Smirnova et al., "Defective LPS signaling in $\mathrm{C} 3 \mathrm{H} / \mathrm{HeJ}$ and $\mathrm{C} 57 \mathrm{BL} / 10 \mathrm{ScCr}$ mice: mutations in Tlr4 gene," Science, vol. 282, no. 5396, pp. 2085-2088, 1998.

[86] H. Duboc, S. Rajca, D. Rainteau et al., "Connecting dysbiosis, bile-acid dysmetabolism and gut inflammation in inflammatory bowel diseases," Gut, vol. 62, no. 4, pp. 531-539, 2013.
[87] S. Oliva, G. Di Nardo, F. Ferrari et al., "Randomised clinical trial: the effectiveness of Lactobacillus reuteri ATCC 55730 rectal enema in children with active distal ulcerative colitis," Alimentary Pharmacology and Therapeutics, vol. 35, no. 3, pp. 327-334, 2012.

[88] W. Kruis, E. Schütz, P. Fric, B. Fixa, G. Judmaier, and M. Stolte, "Double-blind comparison of an oral Escherichia coli preparation and mesalazine in maintaining remission of ulcerative colitis," Alimentary Pharmacology and Therapeutics, vol. 11, no. 5, pp. 853-858, 1997.

[89] B. J. Rembacken, A. M. Snelling, P. M. Hawkey, D. M. Chalmers, and A. T. R. Axon, "Non-pathogenic Escherichia coli versus mesalazine for the treatment of ulcerative colitis: a randomised trial," The Lancet, vol. 354, no. 9179, pp. 635-639, 1999.

[90] W. Kruis, P. Frič, J. Pokrotnieks et al., "Maintaining remission of ulcerative colitis with the probiotic Escherichia coli Nissle 1917 is as effective as with standard mesalazine," Gut, vol. 53, no. 11, pp. 1617-1623, 2004.

[91] D. Jonkers, J. Penders, A. Masclee, and M. Pierik, "Probiotics in the management of inflammatory bowel disease: a systematic review of intervention studies in adult patients," Drugs, vol. 72, no. 6, pp. 803-823, 2012.

[92] J. Shen, Z.-X. Zuo, and A.-P. Mao, "Effect of probiotics on inducing remission and maintaining therapy in ulcerative colitis, Crohn's disease, and pouchitis: meta-analysis of randomized controlled trials," Inflammatory Bowel Diseases, vol. 20, no. 1, pp. 21-35, 2014. 


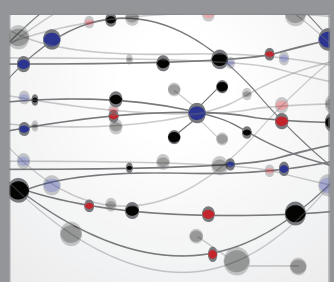

The Scientific World Journal
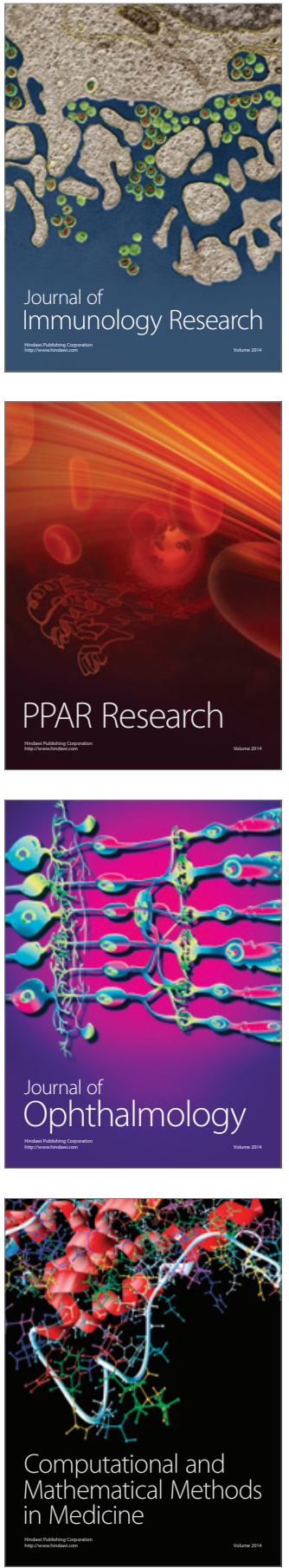

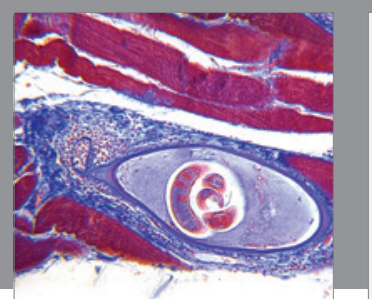

Gastroenterology Research and Practice

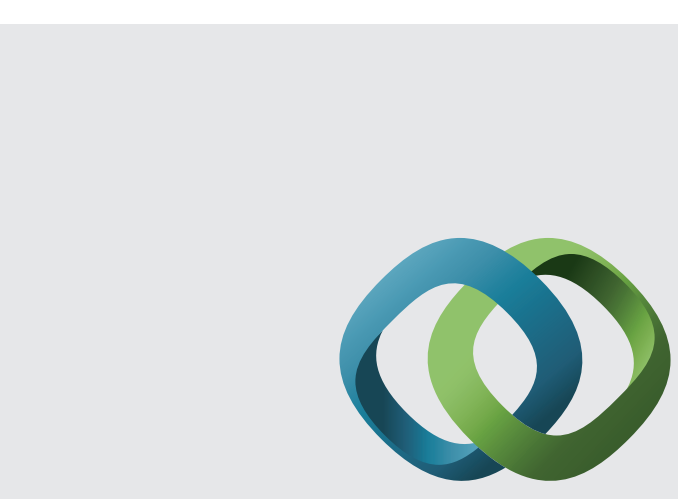

\section{Hindawi}

Submit your manuscripts at

http://www.hindawi.com
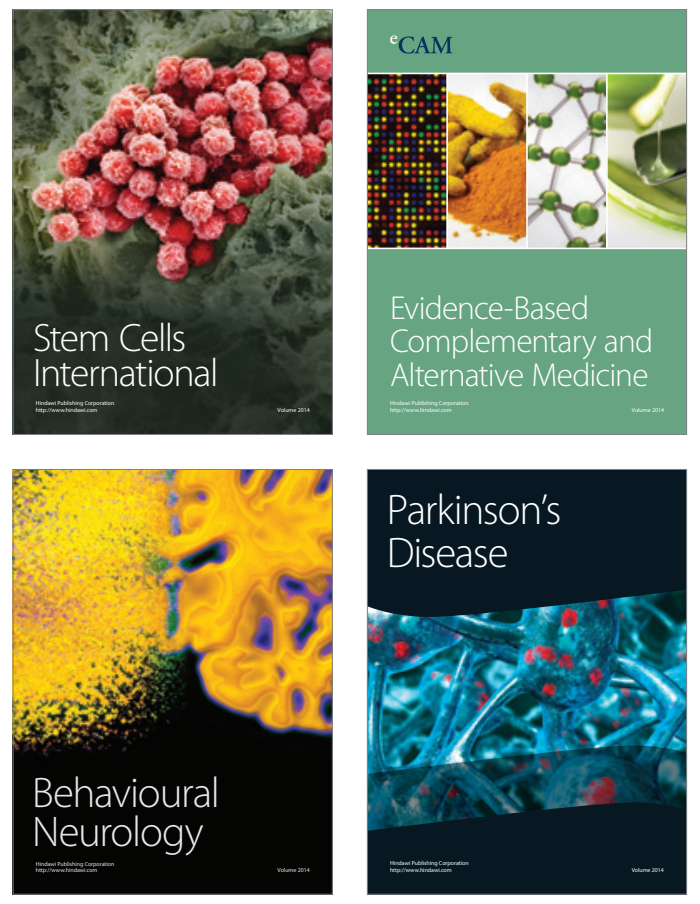
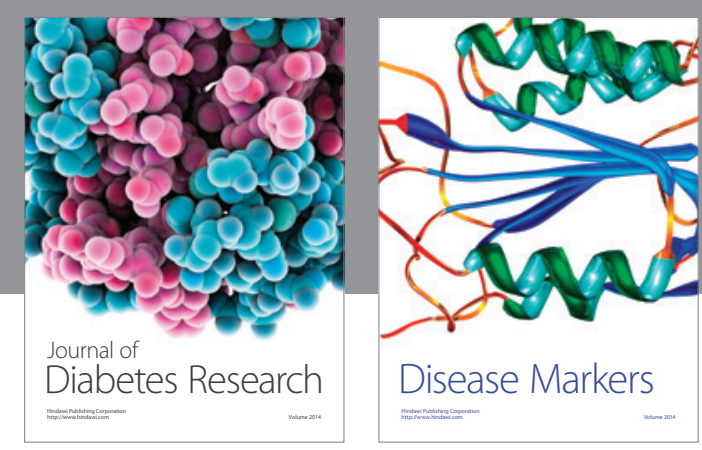

Disease Markers
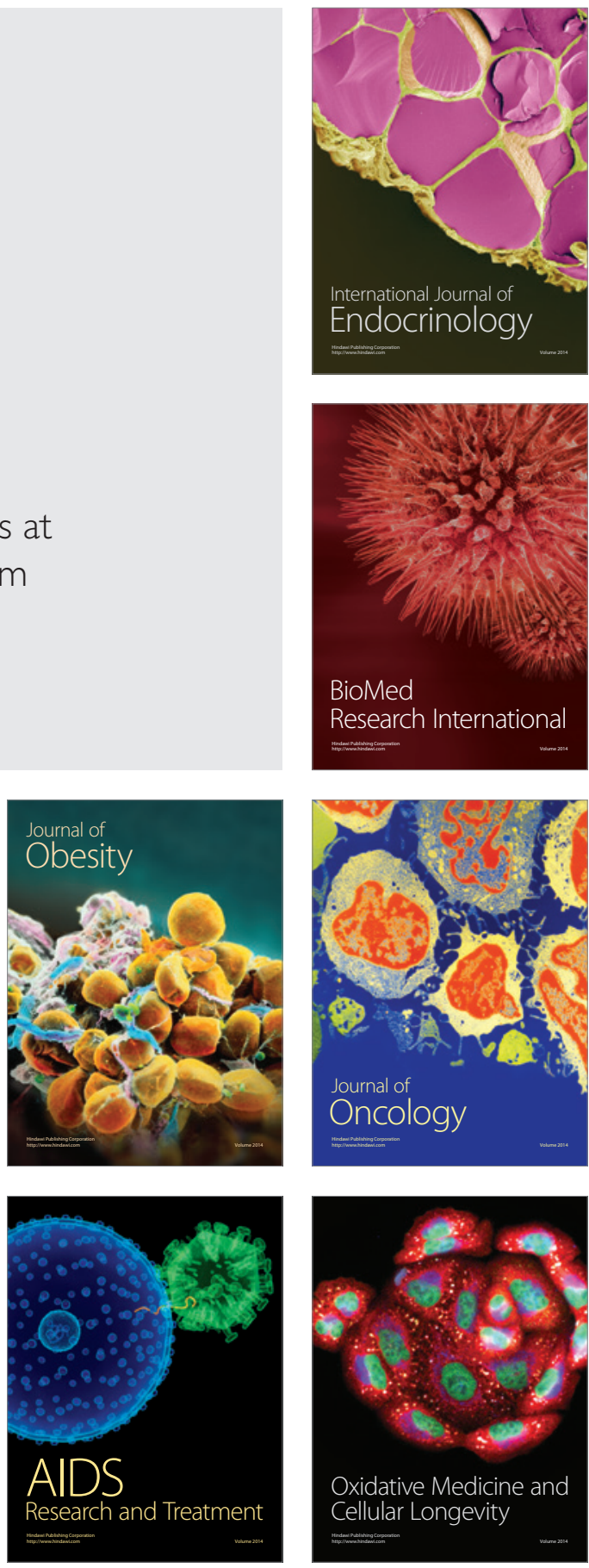